\title{
Levantamento de membrana sinusal com instalação imediata do implante
}

\author{
Sinus lift with immediate implant placement
}

ARAUJO, Felipe Moura ${ }^{1}$; PEDROSA, Rafael Lucas de Oliveira ${ }^{2}$; GHIRALDINI, Bruna ${ }^{3}$; BEZERRA, Fabio Jose Barbosa ${ }^{4}$

1. Doutorando em Implantodontia, Universidade Guarulhos, Guarulhos, SP, Brasil.

2. Especializando em Implantodontia e Prótese, ETEG, Brasil.

3. Doutora em Implantodontia, Universidade Paulista, São Paulo, SP, Brasil.

4. Doutor em Biotecnologia, Instituto de Biociências, Universidade Estadual Paulista, Botucatu, SP, Brasil.

Endereço para correspondência:

Felipe Moura Araujo

Rua Irineu Pinto, 44

Bairro São João Batista

31510-020 - Belo Horizonte - Minas Gerais - Brasil

E-mail:dr.felipemouraaraujo@gmail.com

Recebido: 13.01 .2020

Aceito: 31.03 .2020

\section{RESUMO}

Este estudo tem por finalidade relatar um caso clínico em que a paciente foi submetida a reabilitação com implantes dentários na região posterior de maxila. Para o planejamento do caso, foi feito a anamnese, exames clínicos e imagiológico, que permitiu avaliar o remanescente e o tipo ósseo, fatores determinantes para o sucesso do implante. Foi observada a ausência de osso suficiente para o procedimento, com isso, após estudos e planejamento, optou-se por realizar enxertia óssea e, no mesmo momento cirúrgico, a instalação do implante dentário Strong SW® da S.I.N. Implant System. Tanto o implante escolhido como a técnica para enxerto ósseo foram escolhidos de forma a favorecerem a estabilidade primária do implante, precursor de maior sucesso na osteointegração.

Palavras-chave: Osseointegração. Regeneração óssea. Implantação dentária.

\footnotetext{
ABSTRACT

This study aims to report a clinical case in which the patient underwent rehabilitation with dental implants in the posterior region of the maxilla. For case planning, anamnesis was carried out in addition to clinical and imaging tests, which allow us to assess the remnant and bone type, determining factors for the success of the implant. It was observed the absence of sufficient bone for the procedure, with that, after studies and planning, it was decided to perform bone grafting and, at the same surgical moment, the installation of the S.I.N. Strong SW® dental implant. Implant System. Both the implant chosen and the technique for bone graft were chosen in order to favor the primary stability of the implant, the most successful precursor to osteointegration.
}

Keywords: Osseointegration. Bone regeneration. Dental implantation. 


\section{INTRODUÇÃO}

Com o passar dos anos e o envelhecimento natural, é comum, por diversos fatores, a perca de elementos dentais. A fim de suprir a falta do equilíbrio funcional, fonético e estético, Brånemark, na década de 60 , propôs a ancoragem de próteses dentais sobre pinos metálicos instalados no osso, os implantes dentais1, técnica essa que hoje é consolidada pela literatura e altamente previsível, atingindo taxas de $98 \%$ de sucesso ${ }^{2}$. Entretanto, um fator limitante a ser vencido é a disponibilidade óssea, uma vez que esta sofre com reabsorções e remodelações decorrentes de extrações, traumas, processos patológicos etc..$^{3-4}$.

A perda de uma ou mais unidades dentárias em região posterior de maxila leva a reabsorção óssea e a pneumatização do seio maxilar ${ }^{5}$, o que obriga a se pensar em reabilitações mais complexas que envolvam enxertia óssea na região, para que o implante possa osseointegrar por completo. Esse enxerto é feito pela técnica de levantamento da membrana sinusal, que consiste no descolamento e levantamento da membrana por uma janela lateral e preenchimento do assoalho do seio maxilar com osso autógeno e/ou xenógeno ${ }^{6}$.

A instalação do implante pode ser feita após a cicatrização desse enxerto (de 6 a 8 meses) ou no mesmo momento cirúrgico, o que diminui a morbidade para o paciente por reduzir um tempo operatório, além de encurtar o período de tratamento. Para a escolha da técnica é preciso avaliar a quantidade e a densidade do osso remanescente, visto que esses são fatores determinantes na estabilidade primária do implante, crucial para o sucesso da osteointegração ${ }^{7-8}$.

O emprego correto dos protocolos clínicos nas cirurgias de regeneração, nos permitem ter segurança e previsibilidade no tratamento, tornando essa técnica viável e aumentando as taxas de sucesso.

\section{RELATO DE CASO}

Para este caso foi selecionada uma paciente, do sexo feminino, com queixa a ausência do elemento 14, que incomodava tanto estética como funcionalmente. A mesma possuía uma coroa provisória insatisfatória na região como visto na Figura 1.

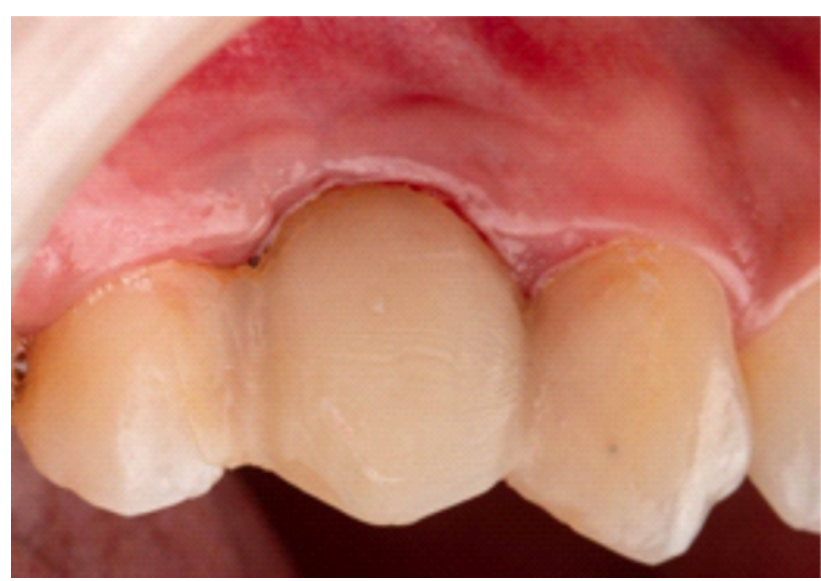

Figura 1 - Coroa provisória.

Após a realização do exame clínico e anamnese, foi solicitado o exame complementar da área a ser reabilitada (tomografia computadorizada).
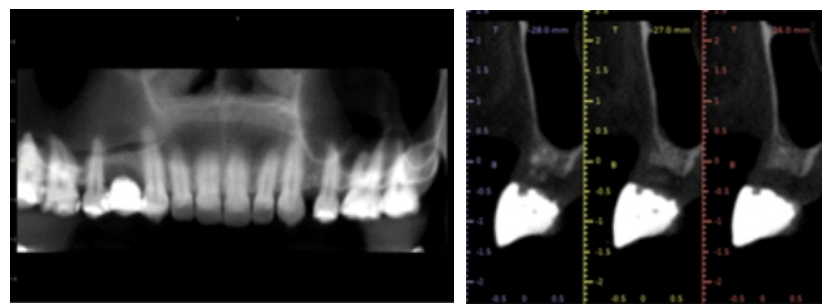

Figuras 2 e 3 - Tomografia computadorizada.

O corte da tomografia nos mostra a situação do rebordo alveolar remanescente da região posterior de maxila. Após a análise e estudo do caso, foi escolhida como forma de tratamento a reabilitação com prótese implantossuportada, sendo o levantamento da membrana sinusal para enxerto ósseo e a instalação do implante realizados no mesmo tempo cirúrgico.

A sequência operatória foi realizada segundo o protocolo da técnica sendo:

(1) Incisão intrassucular com relaxantes na região anterior e descolamento de tecido mole da região.

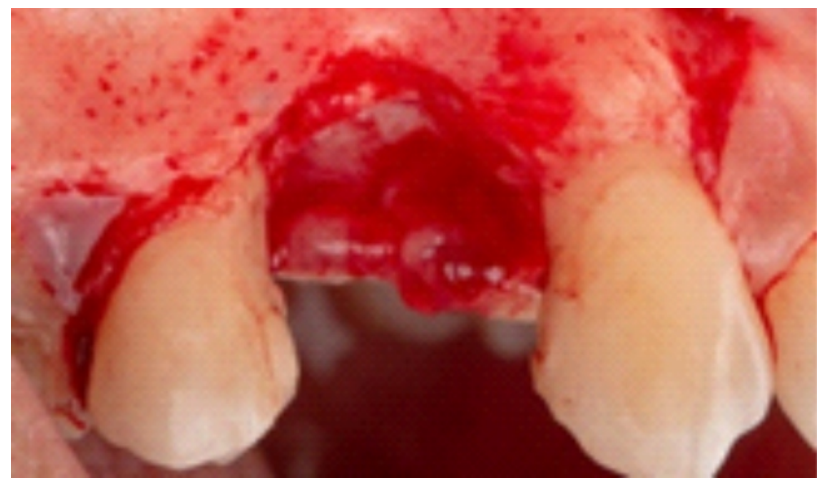

Figura 4 - Incisão intrassucular. 
(2) Abertura da janela cirúrgica utilizando um instrumento de vibração (piezzo) para corte do osso e minimizar as chances de ruptura da membrana sinusal.

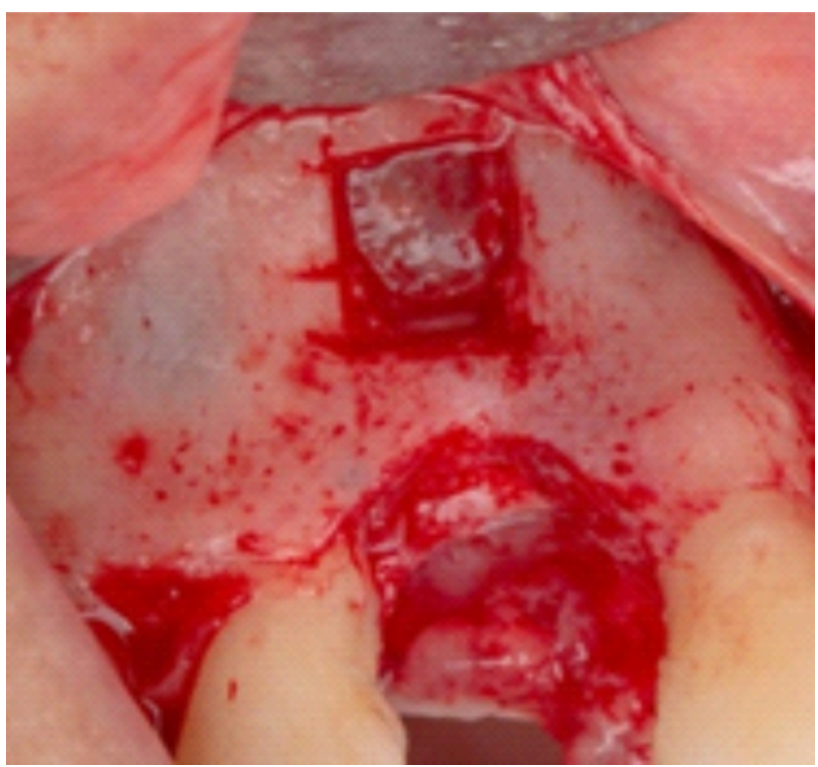

Figura 5-Abertura da janela cirúrgica.

(3) Descolamento e levantamento da membrana sinusal usando curetas do Kit de levantamento de seio (S.I.N. Implant System) que possuem angulações e formatos apropriados para esse procedimento.

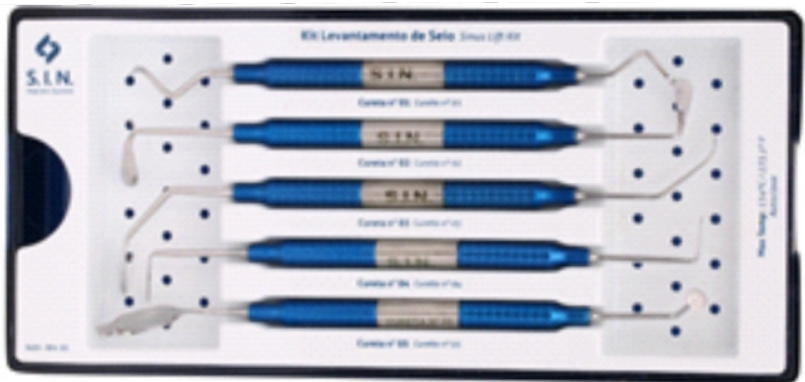

Figura 6 - Curetas do Kit de levantamento de seio.

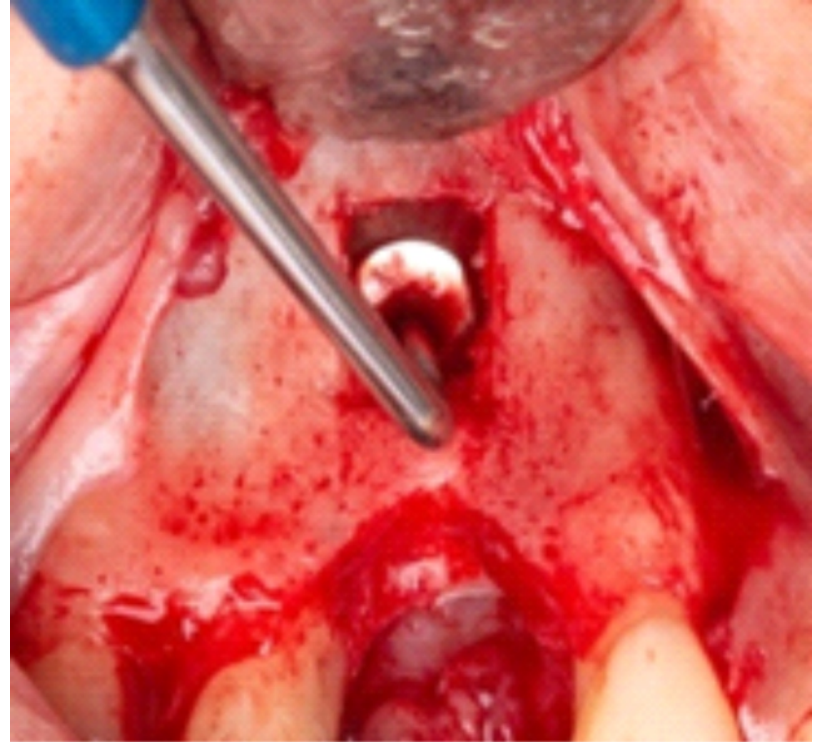

Figura 7 - Membrana sinusal.

(4) Finalizado o descolamento, foi inicializada a sequência de fresagens para instalação do implante (Strong SW ), preconizada no protocolo operatório da S.I.N. Implant System.
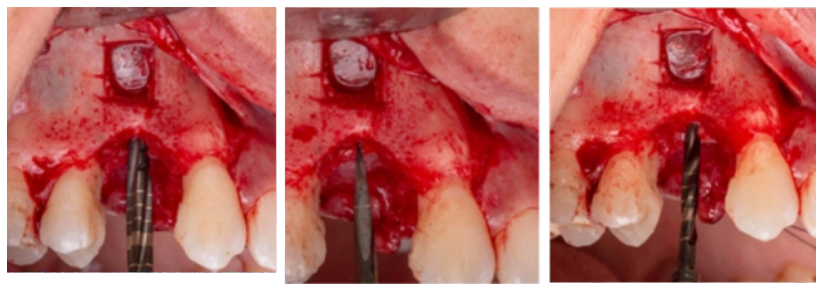

Figuras 8, 9 e 10 - Sequência de fresagens.

(5) Seguido do preenchimento do assoalho do seio maxilar com biomaterial (Bonefil Porus Bionnovation ${ }^{\circledR}$ ) utilicou-se a cureta de $\mathrm{N}^{\circ} 5$ do Kit de levantamento de Seio da S.I.N. Implant System, através da janela lateral que fora previamente aberta.
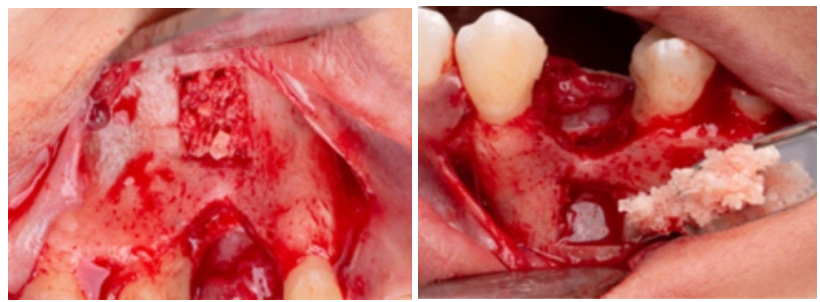

Figuras 11 e 12 - Preenchimento do assoalho do seio maxilar.

(6) Instalação do implante Strong SW. 

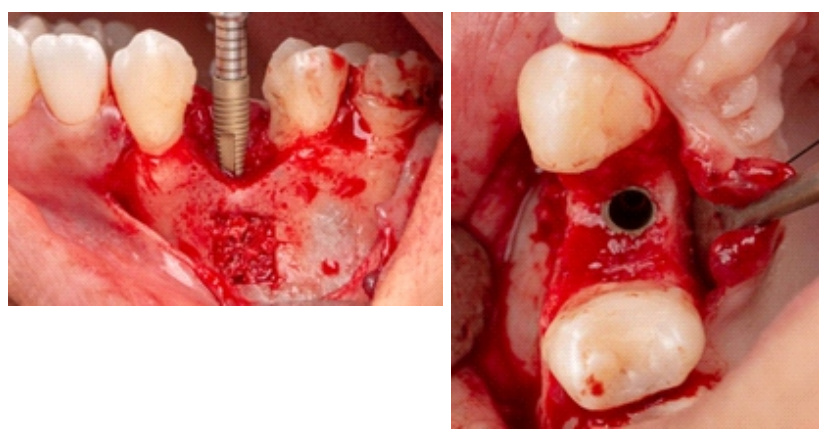

Figuras 13 e 14 - Instalação do implante.

(7) Reposicionamento dos tecidos moles e sutura da ferida cirúrgica.

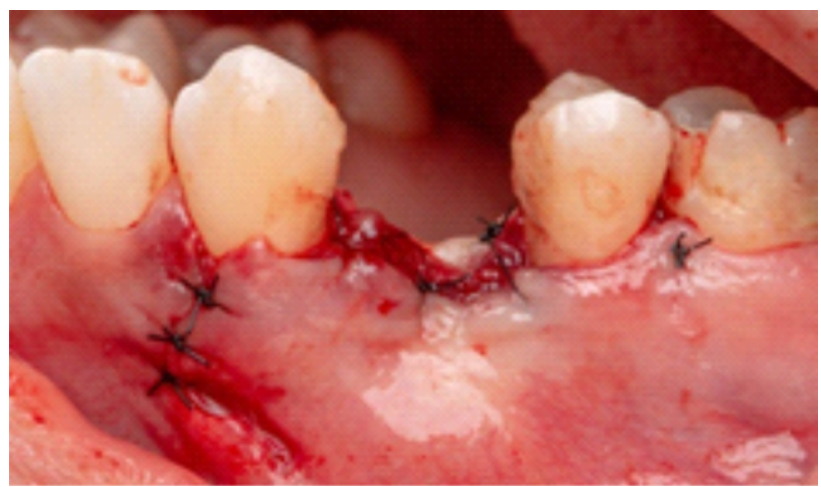

Figura 15 - Reposiconamento e sutura.

\section{DISCUSSÃO}

Para o planejamento voltado para implantes dentários, é fundamental o exame tomográfico, pois o mesmo nos proporciona mensurações mais reais ao comparado com radiografias panorâmicas9. Regiões de maxila posterior, é de suma importância o conhecimento e avaliação da quantidade e qualidade óssea, além da estabilidade primária do implante. Existem quatro tipos de osso (I,II,III e IV) considerando a densidade óssea, sendo o tipo I mais cortical (mais denso) e o tipo IV o mais medular (menos denso). A maxila é composta basicamente por osso tipo III e $\mathrm{IV}$, determinando como fator de sucesso no travamento primário do implante o remanescente ósseo ${ }^{10}$.

Com a finalidade de eleger critérios referentes ao tipo de técnica cirúrgica mais acertivas, uma classificação que considera a altura óssea da maxila posterior foi desenvolvida, a classificação subantral de Misch ${ }^{11}$.

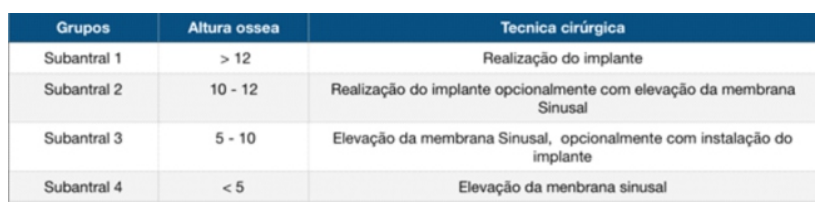

Figura 16 - Classificação subantral de Misch ${ }^{11}$.

Várias técnicas são observadas para acessar e elevar a membrana sinusal, no entanto, duas técnicas se destacam nesse contexto. Uma técnica definida pela elevação lateral com preenchimento de substituto ósseo, seguida a instalação do implante após a cicatrização óssea. Outra técnica da qual, tanto a elevação da membrana sinusal com preenchimento de substituto ósseo, quanto à instalação do implante, são realizados no mesmo momento cirúrgico, em que se tem com pré-requisito, uma quantidade mínima de remanescente ósseo para que seja possível o travamento do implante ${ }^{12}$.

Estudos clínicos sugerem que a colocação de implante simultaneamente com a elevação da membrana sinusal, em rebordos com remanescente ósseo reduzido, pode ser uma modalidade de tratamento viável quando a estabilidade primária adequada do implante pode ser assegurada. As vantagens desta abordagem em um único tempo cirúrgico são a diminuição do tempo de tratamento, a redução do custo e a menor morbidade, pois evita a necessidade de uma segunda cirurgia, a principal desvantagemcom o procedimento de instalação imediata, é a possibilidade de não conseguir estabilizar os implantes em alturas ósseas mínimas, com o risco adicional de os implantes caírem dentro do seio ${ }^{13}$.

A técnica de levantamento da membrana sinusal de forma atraumática, foi descrita primeiro por Tantum e, posteriormente, aprimorada por Summers ${ }^{6,14}$.

A mesma requer uma altura óssea mínima e consiste na utilização de instrumentais que possuem a extremidade côncava, chamados de osteótomos. Ao percurtir os mesmos, com auxilio de um martelo cirúrgico, o osso remanescente é compactado no ápice do alvéolo, até que uma fratura intencional dessa crista óssea ocorra. A pressão exercida pelo material eleva a membrana de Schneiderian, obtendo alguns milímetros a mais para a instalação do implante.

O implante de escolha para o caso reportado foi o Strong SW Plus ${ }^{\circledR}$ da S.I.N. Implant System, devido as suas características de tratamento de superfície e macrogeometria da qual seja possível uma acelerada cicatrização óssea e seguida de um seguro travamento primário.

As modificações nas superfícies dos implantes nas escalas micrométricas e nanométricas tem como objetivo acelerar a resposta óssea e a possibilidade de reabilitação funcional em tempos menores do que aqueles convencionalmente propostos. Estas podem ser de natureza química, por exemplo, incluindo a incorporação de fases inorgânicas sobre ou na camada de $\mathrm{TiO} 2$ com fosfato de cálcio, ou físicas, onde a arquitetura topográfica é alterada ${ }^{15-16}$.

Em caracterizações topográficas da superfície 
nanométrica do implante Strong SW Plus ${ }^{\circledR}$, observa-se a presença de uma superfície inteiramente recoberta por nanocristais de HA com espessura homogênea de $20 \mathrm{~nm}$. A presença da nanotopografia de hidroxiapatita na superfície do implante aumenta marcadores osteogênicos importantes como a fosfatase alcalina e a osteocalcina, além de reduzir a inflamação ${ }^{16}$.

Esse sistema de implante nos traz uma série de benefícios durante o procedimento cirúrgico e no pósoperatório de curto e longo prazo. São eles:

1. A simplificação do procedimento cirúrgico, que requer um menor número de fresas para a instalação do implante e assim garantem menor tempo operatório;

2. A redução do trauma pós-operatório, também de forma associada ao tempo cirúrgico reduzido;

3. Alta estabilidade primária conferida pela forma geométrica do implante e de suas roscas, assim como a utilização de um sistema de fresagem preciso;

4. Manutenção óssea a longo prazo, associadas à conexão interna ser do tipo Cone Morse e a presença de micro-roscas na superfície do implante, essenciais no processo de estabilidade secundária e ajudam na manutenção do osso neoformado;

5. Estabilidade do componente protético devido à melhor dissipação de força conferida pela conexão interna tipo Cone Morse;

6. Plataform switching. Consiste em um distanciamento da interface de conexão pilar/implante. Assim, melhores resultados são atingidos em estética, manutenção de tecido ósseo e saúde do tecido periimplantar ao longo prazo.

\section{CONCLUSÃO}

A viabilidade na realização do levantamento da membrana Sinusal somado à instalação imediata do implante, permitiu redução do tempo de tratamento, conferindo ao paciente maior conforto e menor morbidade, uma vez que se exclui a necessidade de outro processo cirúrgico.

\section{REFERÊNCIAS}

1. Brånemark P-I, Adell R, Breine U, Hansson BO, Lindstrom J, Ohlsson A. Intra-osseous anchorage of dental prostheses. I. Experimental studies. Scand J Plast Reconstr Surg. 1969;3(2):81-100.

2. Wennerberg A, Albrektsson T. Current challenges in successful rehabilitation with oral implants. J Oral Rehabil. 2011;38(4):286-94.

3. Rodrigues CAV. Técnica cirúrgica para elevação do assoalho do seio maxilar: uma revisão de literatura [trabalho de conclusão de curso. Florianópolis: Universidade Federal de Santa Catarina; 2014.
4. Draenert FG, Huetzen D, Neff A, Mueller WE. Vertical bone augmentation procedures: Basics and techniques in dental implantology. J Biomed Mater Res A. 2014;102(5):1605-13.

5. Albuquerque AFM, Cardoso IML, Silva JSP, Germano AR, Dantas WRM, Gondim ALMF. Levantamento de seio maxilar utilizando osso liofilizado associado a instalação imediata de implante do tipo cone morse: relato de caso. RFO. 2014;19(1):129-34.

6. Tantum H. Maxillary and sinus implant reconstructions. Dent Clin North Am. 1986;30:207-29.

7. Javed, F; Ahmed, HB; Crespi, R; Romanos, GE. Role of primary stability for successful osseointegration of dental implants: factors of influence and evaluation. Interv. Med. Appl. Sci. 2013;5:162-7.

8. Kaufman, E. Maxillary sinus elevation surgery: an overview. J Esthet Restor Dent. 2003;15(5):272-83.

9. Héctor González-Santana H, Peñarrocha-Diago M, Guarinos-Carbó J, Sorní-Bröker M. A study of the septa in the maxillary sinuses and the subantral alveolar processes in 30 patients. J Oral Implantol. 2007;33(6):340-3.

10. Fugazzotto PA, Vlassis J. Long-term sucess of sinus augmentation using various surgical approaches and grafting materials. Int J Oral Maxillofac Implants. 1998;13:52-8.

11. Carl EM. Bone density: a key determinant for clinical success. In Contemporary implant dentistry. Dental implant. 2nd ed. Maryland Heights: Mosby; 1999.

12. Boyne PJ, James RA. Grafting of the maxillary sinus floor with autogenous marrow and bone. J Oral Surg. 1980;38:613-6.

13. Cha HS et al. Simultaneous sinus lift and implant installation: prospective study of consecutive two hundred seventeen sinus lift and four hundred sixty-two implants. Clin Implant Dent Relat Res. 2014;16(3):337-47.

14. Summers RB. The osteotome technique part 3: less invasive methods of elevating the sinus floor. Compend Contin Educ Dent. 1994;15:698-708.

15. Coelho, PG, Granjeiro JM, Romanos GE, Suzuki M, Silva NR, Cardaropoli G, et al. Basic research methods and current trends of dental implant surfaces. J Biomed Mater Res B Appl Biomater. 2009;88(2):579-96.

16. Bezerra FJB, Fernando H, Roberto SP, Estevam AB. Unitite ${ }^{\circledR}$ [Internet]. São Paulo: S.I.N. Implant System, 2020 [cited 2020 Jan 6]. Ava i l a ble from: https://www.sinimplantsystem.com.br/produto/unitite/. 